\title{
Preservation of tangent modulus of pericardial tissue during cold storage
}

\author{
E.Gultova* ${ }^{*}$, L.Horny $\dagger$, H. Chlup $\dagger$, R. Zitny $\dagger$, T. Adamek $\ddagger$ and Marketa Kulvajtova ${ }^{+}$ \\ † Faculty of mechanical Engineering, Czech Technical University in Prague, Technicka 4,16607 Prague \\ ¥ Third Faculty of Medicine, Charles University, Ruska 87, 10000 Prague \\ ${ }^{+}$University Hospital Na Kralovskych Vinohradech, Srobarova 1150/50, 10034 Prague
}

Keywords: human pericardium; storage effect; tangent modulus; biomechanics.

\section{Introduction}

The significance of studying of human pericardial mechanics arises from a wide range of possible applications. For instance, tissue engineers study potential use of a human pericardium in a design of homograft valve bioprosthesis. Scientists deal with different mechanical properties, effect of chemical treatment, impact of loading history etc. Current scientific literature does not contain enough experimental data to evaluate usable life of the pericardial tissue so the question of the influence of the storage time on the mechanical properties is still open.

The goal of this pilot study is to determine changes in passive mechanical response of the human pericardium, based on results of uniaxial testing, as a consequence of material storage.

\section{Methods}

Eight samples of the human pericardium dissected from male cadaver (age 33) were prepared for the purpose of the mechanical testing. Respecting anisotropy of material, specimens were cut in the direction of heart meridian and circumference (Figure 1).

Samples were dissected in the autopsy with the approval of the Ethic Committee of the University Hospital Na Kralovskych Vinohradech in Prague. The specimen dimensions were measured by Laser profile sensor ScanControl 2800 (Micro-Epsilon, Ortenburg, Germany) and then the pericardial tissue was stored in a $5^{\circ} \mathrm{C}$ refrigerator in a distilled water. Experiments were conducted after storage for 30 , 54, 76 and 100 hours.

Uniaxial tensile tests were performed on the customer specific biaxial testing machine (Zwick/Roell). Experiments were recorded by a videoextensometer which provides for online detection of the deformation. The tests were controlled according following scheme: four cycles of loading with a constant limit deformation (for preconditioning of material) followed by stress relaxation, were completed by fifth loading cycle till a failure.

The mechanical response was modeled as incompressible and hyperelastic. Using Fung's exponential form of the strain energy density function and assuming isotropic deformation, Cauchy stress is expressed (Demiray, 1972):

$$
\sigma=\mu \cdot \mathrm{e}^{C \cdot\left(\lambda^{2}+\frac{2}{\lambda}-3\right)} \cdot\left(\lambda^{2}-\frac{1}{\lambda}\right),
$$

where $\mu$ represents stress-like parameter (corresponding with initial infinitesimal shear modulus), $c$ is dimensionless parameter and $\lambda$ is stretch (defined as ratio of actual and referential length) in the direction of loading.

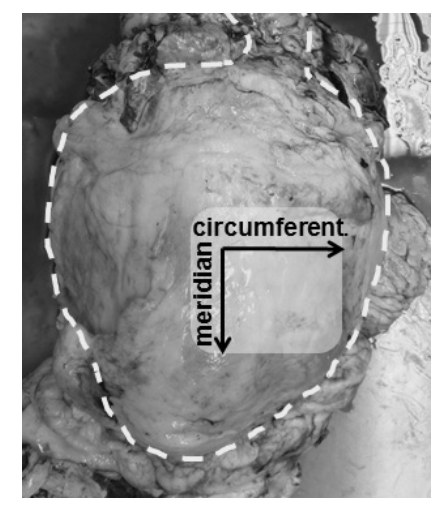

Figure 1 Circumferential and meridian orientation of the pericardial samples.

\section{Results and Discussion}

The nonlinear response of the human pericardium is depicted in the Figure 2.

Material parameters $\mu$ and $c$ of the model (1) are described in Table 1 . Although $\mu$ and $c$ vary between samples of different PMI, there is no characteristic trend.

The stress-stretch curves (Figure 2) in initial deformation differ not significantly contrary to the mechanical response corresponding with higher deformation $(\lambda>1.05)$. The longer PMI, the higher stress response at particular stretch, nevertheless only limiting change in tangent of the stress-stretch curve depending on the PMI was observed. The decrease in mechanical response was observed according Wagenseil et al. (2011) after 14 days however this storage range was not applied in our preliminary study. 


\begin{tabular}{|c|c|c|}
\hline Sample & $\mu[\mathrm{MPa}]$ & $c[-]$ \\
\hline C30 & 2.7 & 82 \\
\hline C54 & 2.6 & 102 \\
\hline C76 & 3.5 & 78 \\
\hline C100 & 3.4 & 85 \\
\hline \hline M30 & 9.8 & 53 \\
\hline M54 & 7.8 & 95 \\
\hline M76 & 10.3 & 77 \\
\hline M100 & 10.2 & 84 \\
\hline
\end{tabular}

Table 1 Material parameters of each specimen. The samples are labeled according rule: $\mathrm{C} / \mathrm{M}$ denotes cut orientation (circumferential/meridian) and 30/54/76/100 means post mortem interval in hours.

Preservation of mechanical response after storage of the samples in refrigerator is not in agreement with expected behavior connected with decomposition of a cadaver tissue. The problematic could be clarified by chemical analysis of the putrefied tissues. The results indicate that processes decomposing components responsible for mechanical stability could be decelerated.

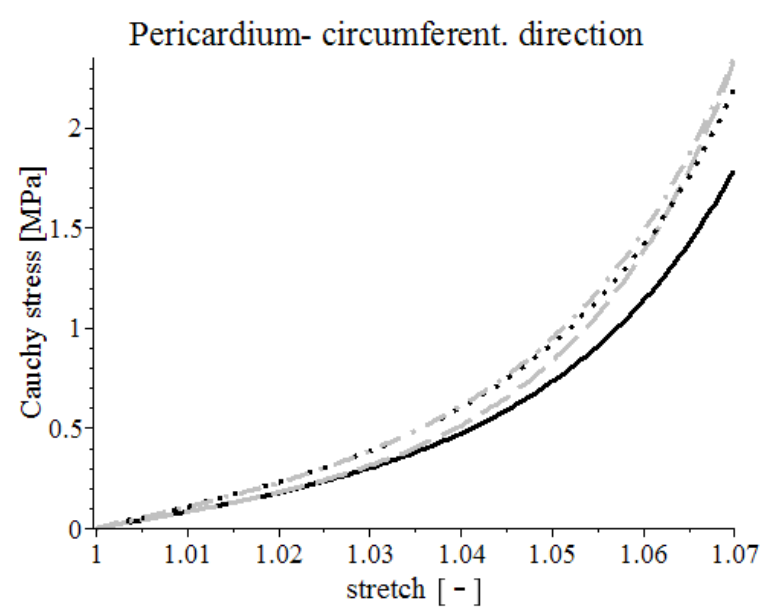

Pericardium - meridian direction

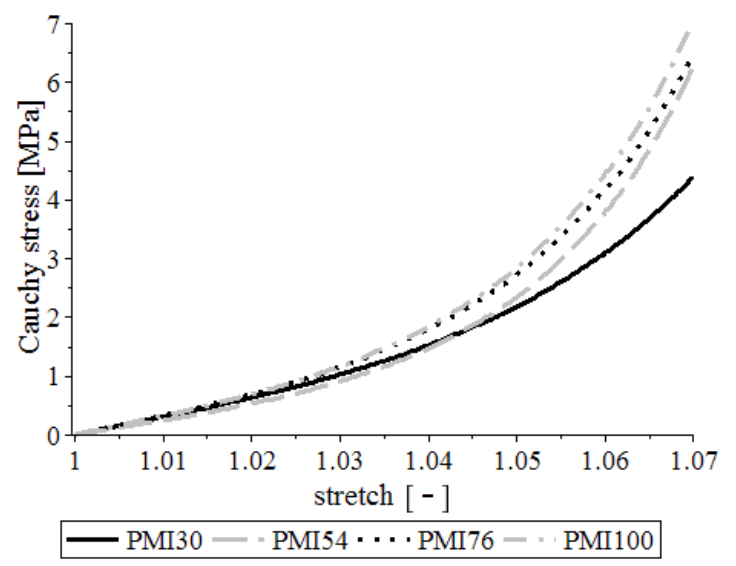

Figure 2 Cauchy stress-stretch response of material at different $\mathrm{PMI}=$ post mortem interval in hours (=storage time). Upper/lower figure represents circumferential/ meridian samples.

\section{Conclusions}

We have performed uniaxial tensile tests with human pericardium in order to identify the effect of a storage time on the passive mechanical response. Mechanical response was modeled using simple Fung's type form of the strain energy density function, assuming isotropic hyperlestic response. The final stress-stretch curves indicate that primary mechanical response could be hold for a few days post mortem. Our results are nevertheless got from limited number of samples from only one cadaver. In the case that the following tests confirm that the mechanical properties of a cadaver tissue does not change during 100 hours, new opportunities in the field of tissue-engineering dealing with homograft valve bioprosthesis could be opened.

\section{Acknowledgments}

This work has been supported by the Grant Agency of the Czech Technical University in Prague, grant No. SGS10/247/OHK2/3T/12.

\section{References}

Demiray H. 1972. A note on the elasticity of soft biological tissues. J. Biomech. 5:309-311.

García Páez JM, Jorge Herrero E et all.2003. Comparison of the mechanical behaviors of biological tissues subjected to uniaxial tensile testing: pig, calf and ostrich pericardium sutured with Gore-Tex. Biomaterials. 24/9:1671-1679.

Sacks MS, Hamamoto H, Connolly JM et all. 2007. In vivo biomechanical assessment of triglycidylamine crosslinked pericardium. Biomaterials. 28/35:5390-5398.

Wagenseil JE, Amin M, Kunkel AG, Le VP. 2011. Effect of storage duration on the mechanical behavior of mouse carotid artery. Journal of Biomechanical Engineering. 133:071007-1071007-6.

Zioupos P, Barbenel JC. 1994. Mechanics of native bovine pericardium, I The multiangular behavior of strength and stiffness of the tissue. Biomaterials. 15/5: 366-373.

Zioupos P, Barbenel JC. 1994. Mechanics of native bovine pericardium,II A structure based model for the anisotropic mechanical behaviour of the tissue. Biomaterials. 15/5: 374-382. 